\title{
Eosinophilic myelodysplasia transforming to acute lymphoblastic leukaemia
}

\author{
G A Follows, R G Owen, A J Ashcroft, L A Parapia
}

\begin{abstract}
A 43 year old male presented with a marked eosinophilia and associated systemic symptoms. A diagnosis of myelodysplasia was made on the basis of bone marrow morphology and karyotype. Over a 12 month period the disease transformed into acute lymphoblastic leukaemia, confirmed by flow cytometry, cytochemistry, and immunohistochemistry. Karyotyping was abnormal with 5qand -7 which persisted from diagnosis through to blastic transformation. He died following initial induction chemotherapy. Eosinophilic myelodysplasia is an uncommon condition in haematological practice and no previous report of lymphoblastic transformation has been found. (f Clin Pathol 1999;52:388-389)
\end{abstract}

Keywords: eosinophilic myelodysplasia; acute lymphoblastic leukaemia

\section{Initial presentation and investigations}

Department of
Haematology,
Bradford Royal
Infirmary, Duckworth
Lane, Bradford
BD9 6RJ UK
G A Follows
R G Owen
A J Ashcroft
L A Parapia

Correspondence to: Dr Owen.

Accepted for publication 29 January 1999

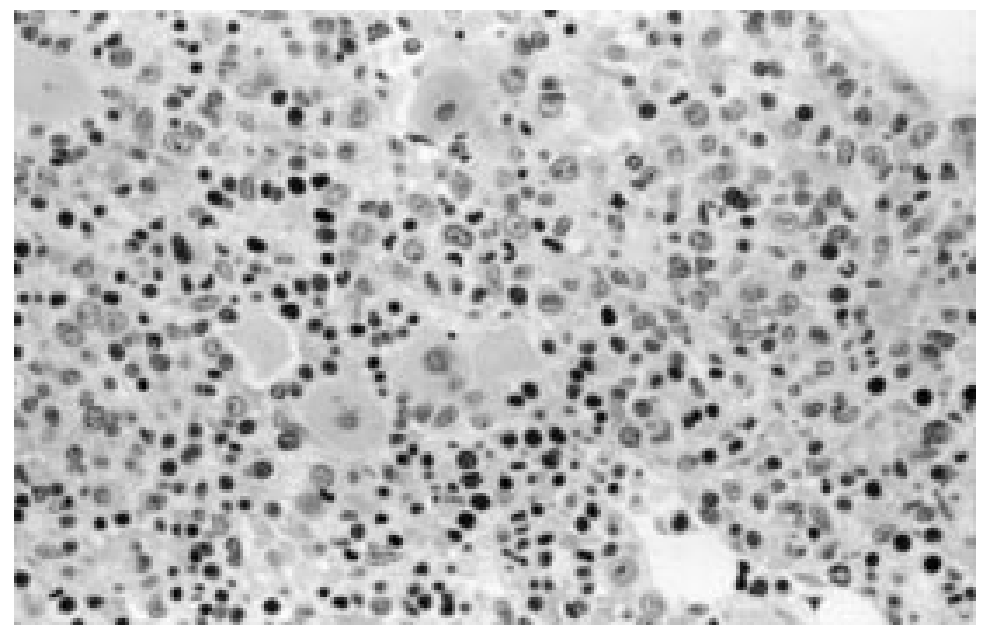

Figure 1 Bone marrow trephine biopsy at diagnosis revealing marked trilineage dysplasia characteristic of myelodysplasia.

A 43 year old male was referred with a two week history of worsening Raynaud's phenomenon and a migratory arthralgia affecting both large and small joints. This was associated with periarticular soft tissue swellings. He had a past history of depression but little else of note, and on admission was taking dothiepin and paracetamol. On examination he had mild hepatosplenomegaly with erythematous tender swollen areas around his left elbow and on the dorsum of his right foot. Otherwise, he had no clinical evidence of lymphoma or of pulmonary or myocardial disease.
Initial investigations revealed an eosinophil count of $13.4 \times 10^{9} /$ litre with normal neutrophil, lymphocyte, monocyte, and platelet counts. A blood film showed dysplastic eosinophils with degranulation but no blast cells. Haemoglobin was $12.9 \mathrm{~g} / \mathrm{dl}$ and a clotting screen was normal. Initial biochemical screen was normal apart from a mildly raised $\gamma$-glutamyl transferase (67 IU/litre). Abdominal ultrasound showed hepatomegaly with a homogeneous increase in echogenicity and an $18 \mathrm{~cm}$ spleen. Subsequent liver biopsy revealed a moderate steatosis with variable eosinophil infiltrate. Skin biopsy showed a similar eosinophil infiltrate in the deeper dermis, particularly around the sweat glands. Bone marrow trephine biopsy (fig 1) revealed a marked increase in cellularity with loss of normal architecture and disruption of normal erythroid colonies with striking dyserythropoiesis. Megakaryocytes were reduced and morphologically atypical, while the myeloid series was expanded and markedly left shifted. Chromosomal analysis revealed a karyotype 45, XY, del(5)(q22q33), $-7, \operatorname{add}(12)(\mathrm{p} 13) .[29] / 46 \mathrm{XY}[1]$.

\section{Management and clinical course}

A diagnosis of myelodysplasia with eosinophilia was made and in view of his systemic symptoms, he was treated initially with hydroxyurea. There was an encouraging early response, but joint stiffness and flitting rashes continued to trouble him. These symptoms partially responded to intravenous steroid treatment, but following a dramatic rise in eosinophils to over $50 \times 10^{9} /$ litre, $\alpha$ interferon (interferon alfa) $3 \mathrm{MU} /$ day was started. Bone marrow examination at this stage showed little change and the same karyotype persisted: 45 , XY, del(5)(q22q33), -7, add(12)(p13).[4]/46 $\mathrm{XY}[1]$. The clinical response to $\alpha$ interferon was initially excellent with almost complete resolution of his multiple symptoms accompanying a fall in his eosinophil count to less than $1 \times 10^{9} /$ litre. Over the next two months symptoms returned, accompanied by an increasing eosinophilia. The interferon dose was increased to $5 \mathrm{MU} /$ day and subsequently hydroxyurea added. Unfortunately all treatment had to be withdrawn as the patient became pancytopenic, requiring red cell and platelet transfusions. Withdrawal of treatment led to a transient increase in cell counts before pancytopenia became established. Repeat bone marrow examination showed the marrow to be almost completely replaced by poorly differentiated blasts characterised by highly irregular nuclei and small nucleoli (fig 2). Cytochemistry showed the blasts to be negative for 


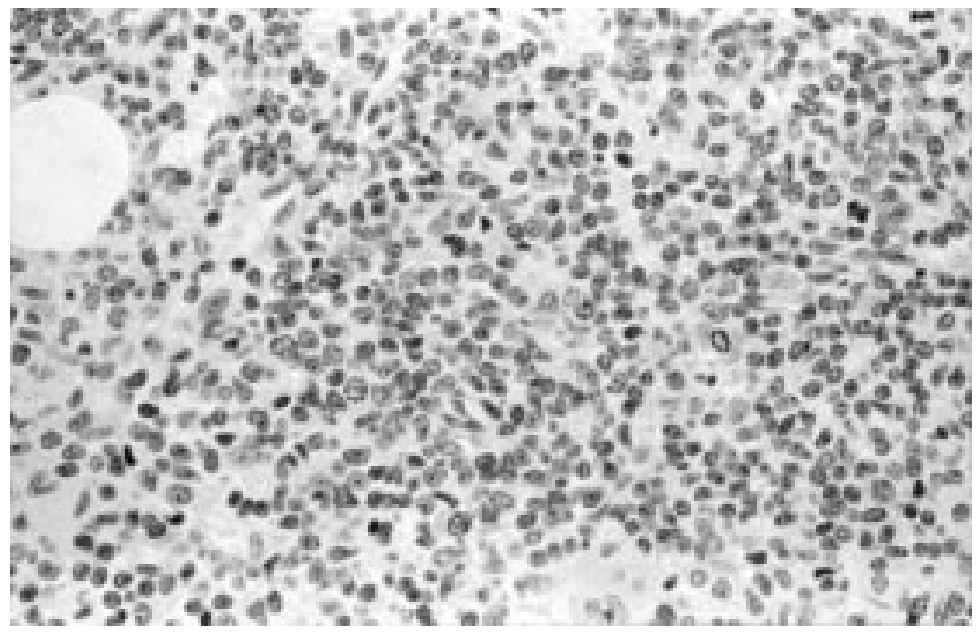

Figure 2 Sheets of poorly differentiated lymphoid blasts in the trephine biopsy at leukaemic transformation. in our patient are, however, typically associated with myelodysplastic syndromes. In addition, the morphological findings on bone marrow examination makes the diagnosis of myelodysplasia with eosinophilia possibly more appropriate. This is, however, not entirely clear cut, as monosomy 7 and chromosome 5 abnormalities have been reported in eosinophilic leukaemia. ${ }^{2}$ While such chromosome 5 abnormalities are usually part of the $\mathrm{t}(5 ; 12)$ translocation, some of these patients have also had evidence of trilineage dysplasia.

Acute lymphoblastic leukaemia complicated by eosinophilia and associated end organ damage is a rare but well documented condition. ${ }^{4}$ It is usually $\mathrm{T}$ cell acute lymphoblastic leukaemia and the eosinophilia is usually reactive, only occasionally being shown to be clonal. There have been reported cases of idiopathic hypereosinophilic syndrome terminating in acute lymphoblastic leukaemia, ${ }^{56}$ but direct clonal links were not demonstrated in these cases. This suggests that these initial eosinophilias may have been reactive prodromal events rather than being clonally related to the acute lymphoblastic leukaemia.

To our knowledge, there have been no reports of eosinophilic myelodysplasia transforming to acute lymphoblastic leukaemia. It is indeed very rare for $5 \mathrm{q}-,-7$ cytogenetic abnormalities to be found in patients with acute lymphoblastic leukaemia, yet these abnormalities persisted from diagnosis to final transformation. This acute transformation is reminiscent of lymphoblastic transformation of $\mathrm{Ph}+$ chronic myeloid leukaemia and presumably reflects the stem cell nature of the underlying disease process. The poor outcome for our patient is, unfortunately, in keeping with other experiences. A study of de novo myelodysplasia patients found that those with associated eosinophilia had a significantly shortened median survival, even in the absence of leukaemic transformation. $^{7}$

We are grateful to the Haematological Malignancy Diagnostic Service (HMDS) at Leeds General Infirmary, Leeds, UK, for performing the immunophenotyping on all samples.

\section{Discussion}

It is not always simple to distinguish between a true myelodysplastic syndrome and eosinophilic leukaemia. In this case the situation is more complex in that the disease behaved in some ways more like a myeloproliferative disorder. Transformation of myelodysplasia into acute lymphoblastic leukaemia is particularly rare but in this case the cytogenetic investigations show that the acute lymphoblastic leukaemia did arise from a clone of abnormal cells that was detected at the original presentation.

It has recently been suggested that eosinophilic syndromes with a clonal cytogenetic abnormality are best classified as eosinophilic leukaemia. ${ }^{1}$ The $5 \mathrm{q}$ - and -7 abnormalities seen
1 Bain BJ. Eosinophilic leukaemias and the hypereosinophilic syndrome. Br F Haematol 1996;95:2-9.

2 Viniou N, Yataganas X, Abazis D, et al. Hypereosinophilia associated with monosomy 7. Cancer Genet Cytogenet 1995; 80:68-71.

3 Keene P, Mendelow B, Pinto MR, et al. Abnormalities of chromosome $12 \mathrm{p} 13$ and malignant proliferation of eosinophils: a non random association. Br f Haematol eosinophils: a

4 Troxell ML, Mills GM, Allen RC. The hypereosinophilic syndrome in acute lymphoblastic leukaemia. Cancer 1984; 54:1058-61

5 Takai K, Sanada M. Hypereosinophilic syndrome evolving to acute lymphoblastic leukaemia. Int f Haematol 1991;54: 231-9.

6 Blatt J, Proujansky R, Horn M, et al. Idiopathic hypereosinophilic syndrome terminating in acute lymphoblastic leukaemia. Pediatr Hematol Oncol 1992;9:151-5.

7 Matsushima T, Murakami H, Sawamura M, et al. MyelodBrf Haematol 1993;84:636-8. 